\title{
2D-HELS-AA MS Seq: Direct sequencing of tRNA reveals its different isoforms and multiple dynamic base modifications
}

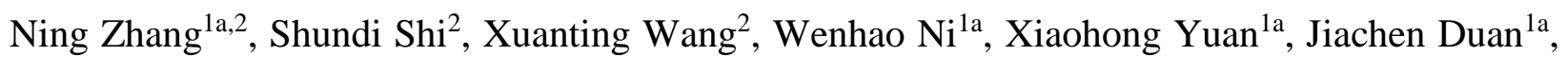
Tony Z. Jia ${ }^{3,4}$, Barney Yoo $^{5}$, Ashley Ziegler ${ }^{1 \mathrm{a}}$, James J. Russo ${ }^{2}$, Wenjia Li ${ }^{1 \mathrm{~b}}$, Shenglong Zhang ${ }^{\mathrm{a} *}$

${ }^{1 a}$ Department of Biological and Chemical Sciences, New York Institute of Technology, New York, NY, 10023, USA.

${ }^{1 b}$ Department of Computer Science, New York Institute of Technology, New York, NY, 10023, USA.

${ }^{2}$ Department of Chemical Engineering, Columbia University, New York, NY, 10027, USA.

${ }^{3}$ Earth-Life Science Institute, Tokyo Institute of Technology, Meguro-ku, Tokyo, 152-8550, Japan.

${ }^{4}$ Blue Marble Space Institute of Science, Seattle, WA, 98154, USA.

${ }^{5}$ Department of Chemistry, Hunter College, City University of New York, New York, NY, 10065, USA.

*Corresponding author: Shenglong Zhang. Email: szhang21@ nyit.edu

Abstract: We report a direct method for sequencing $\mathrm{tRNA}^{\text {Phe }}$ without cDNA by combining 2dimensional hydrophobic RNA end-labeling with an anchor-based algorithm in mass spectrometry-based sequencing (2D-HELS-AA MS Seq). The entire $\mathrm{tRNA}^{\text {Phe }}$ was sequenced and the identity, location and abundance of all 11 base modifications were determined. Changes in ratios of wybutosine and its depurinated form under different conditions were quantified, pointing to the ability of our technology to determine dynamic changes of nucleotide modifications. Two truncated isoforms at $3^{\prime} \mathrm{CCA}$ tail of the $\mathrm{tRNA}^{\text {Phe }}(75 \mathrm{nt} \mathrm{CC}, 80 \%$ and $74 \mathrm{nt} \mathrm{C}, 3 \%)$ were identified in addition to the $76 \mathrm{nt}$ tRNA ${ }^{\text {Phe }}$ with a full-length $3^{\prime} \mathrm{CCA}$ tail $(17 \%)$. We also discovered a new isoform with A-G transitions at both the 44 and 45 positions in the tRNA ${ }^{\text {Phe }}$ variable loop.

One Sentence Summary: Direct 2D-HELS-AA MS Seq of tRNA reveals different isoforms and base modifications

As an essential component of protein synthesis machinery, transfer RNA (tRNA) is present in all living cells and more than 600 different tRNA sequences and a large breadth of different posttranscriptional base modifications that have been reported $(1,2)$. Despite its significance, structural and functional studies to understand the underlying biochemistry of tRNA itself have been hindered due to the lack of efficient tRNA sequencing methods. Although the first tRNA was sequenced, thanks to a herculean effort, by Holley in 1965 (3), tRNAs are currently the only class of small cellular RNAs that cannot be efficiently sequenced with current sequencing techniques (4). Typically, methods used to sequence tRNAs are indirect and require complementary DNA (cDNA) intermediates. However, cDNA synthesis results in a loss of endogenous base modification information originally carried by RNAs, resulting in the inability to accurately sequence the rich and dynamic base modifications in tRNAs which are an intrinsic part of their structure and function. Other sequencing methods that do not involve cDNA can detect base modifications, but these techniques usually require harsh sample treatments such as intensive enzymatic or chemical hydrolysis, resulting loss of in spatial modification information (5-7). As 
opposed to indirect sequencing methods, direct sequencing of RNAs without cDNA synthesis would theoretically allow identification of all associated modified nucleotides in any RNA sample.

One potential direct RNA sequencing method is mass spectrometry (MS)-based sequencing, which directly correlates the mass of each base, rather than proxies such as fluorescence (8) or fluctuations in electric current (9) to sequence outputs. The four canonical ribonucleotides and most modified bases either have inherently unique masses or can be easily converted into different signature masses, allowing them to potentially be used for de novo MS-based direct RNA sequencing. Previously, top-down MS and tandem MS (10-12) have been used in attempts to directly sequence tRNAs. However, these traditional MS methods have significant methodological inadequacies in the preparation of high-quality sequence ladders and subsequently in the reduction of complicated data, resulting in inaccurate base-calling $(13,14)$. Therefore, a two-dimensional (2D) LC-MS-based RNA sequencing method was established to produce easily-identifiable massretention time $\left(\mathrm{t}_{\mathrm{R}}\right)$ ladders (14), allowing de novo sequencing of short single-stranded RNAs. We recently introduced a hydrophobic end-labeling strategy (HELS) to 2D LC-MS-based RNA sequencing by introducing $2 \mathrm{D}$ mass- $\mathrm{t}_{\mathrm{R}}$ shifts for ladder identification of mixed RNAs (15), allowing both the complete reading of a sequence from a single ladder and the sequencing of mixed RNA samples. Despite its success in model studies on short RNAs ( $20 \mathrm{nt})$ (15), it is still challenging to perform de novo MS sequencing of biological RNA such as tRNAs due to the data complexity caused by the increased length (typically 76-90 nt) and abundant modifications. To simplify the 2D HELS data analysis, here we developed two computational anchor algorithms which innovatively accomplish automated sequencing of RNAs. The signature $t_{R}-$ mass value of the hydrophobic tag specifies the exact starting data point, the anchor, for the algorithm to accurately determine data points corresponding to the desired ladder fragments, significantly simplifying data reduction and enhancing the accuracy of sequence generation. The idea of using an anchor to identify sequence ladder start-points can be generalized and extended to any known chemical moiety beyond hydrophobic tags, e.g., $\mathrm{PO}_{4}^{-}$at the beginning of the tRNA or any nucleotide with a known mass. We can program its mass as a tag mass and use our anchor algorithms for sequencing, addressing the issue of MS data complexity and making 2D HELS MS Seq more robust and accurate.

As it was possible to read segments of up to $35 \mathrm{nt}$ long with a $40 \mathrm{~K}$ mass resolution LC-MS (15), we incorporated a partial RNase T1 digest step to sequence a tRNA ${ }^{\text {Phe }}$ that was commercially available, resulting in a reduction of the $76 \mathrm{nt}$ tRNA to fragments of sequenceable sizes. Subsequently, we directly sequenced the entire tRNA with single-base resolution in one single LC-MS run (Fig. 1). To further verify the complete tRNA sequence obtained from the single run above, we labeled the three segments cut by RNase T1 and separated them one by one for 2DHELS-AA (anchor-based algorithm) MS Seq in three separate LC-MS runs (Fig. S1). To obtain overlapping segment sequences for assembling the complete tRNA, we also included MS data of the tRNA generated without RNase T1 digestion (Fig. 1B). Taking all draft reads output by the anchor-based algorithm together (Table S1-11), we assembled a full length tRNA sequence which was a $100 \%$ match to the tRNA ${ }^{\text {Phe }}$ reference sequence with more than $2 x$ coverage (Fig. $1 \mathrm{~B}$ ). We also successfully identified and located all 11 RNA modifications within the tRNA (Fig. 2). Four of these modifications could be directly read out by their unique masses: dihydrouridine (D) at positions 16 and $17, \mathrm{~N}^{2}, \mathrm{~N}^{2}$-dimethylguanosine $\left(\mathrm{m}_{2}^{2} \mathrm{G}\right)$ at position 26,5 -methylcytidine $\left(\mathrm{m}^{5} \mathrm{C}\right)$ at position 40 and 5-methyluridine (T) at position 54. Methylation on the $2^{\prime}-\mathrm{OH}$ of $\mathrm{C}(\mathrm{Cm})$ at position 32 and $\mathrm{G}(\mathrm{Gm})$ at position 34 renders the adjacent $3^{\prime}-5^{\prime}$-phosphodiester linkage non-hydrolyzable, 
creating a mass gap in both the $5^{\prime}$ - and the $3^{\prime}$-mass ladder families larger than $1 \mathrm{nt}$ (14) (Fig. 1A). This gap can be filled by collision induced dissociation (CID) MS, which determines which of the two unhydrolyzable nucleotides is methylated (14) (Fig. 2C and Fig. S2). However, other RNA modifications such as pseudouridine $(\psi)$ and $\mathrm{U}, \mathrm{N}^{2}$-methylguanosine $\left(\mathrm{m}^{2} \mathrm{G}\right)$ and 7methylguanosine $\left(\mathrm{m}^{7} \mathrm{G}\right)$, and 1-methyladenosine $\left(\mathrm{m}^{1} \mathrm{~A}\right)$ and $\mathrm{N}^{6}$-methyladenosine $\left(\mathrm{m}^{6} \mathrm{~A}\right)$ share an identical mass, and LC-MS alone cannot distinguish them. Further enzymatic/chemical reactions were required to differentiate $\mathrm{m}^{1} \mathrm{~A}$ and $\mathrm{m}^{6} \mathrm{~A}(16), \mathrm{U}$ and $\psi$ (15) (Tables S12-17), and $\mathrm{m}^{2} \mathrm{G}$ and $\mathrm{m}^{7} \mathrm{G}$ (17), as shown in the Fig. 2C. Percentages of all the RNA modifications were quantified at each position in case they are not $100 \%$ modified (Fig. 2C).

Upon analysis of the sequencing results, we noticed that the wybutosine (Y) at position 37 was converted to its depurinated product $\mathrm{Y}^{\prime}$ (ribose) under acidic degradation conditions (Fig. 2) (18, 19). Without acid degradation, only $10 \%$ of the tRNA contained the depurinated $\mathrm{Y}^{\prime}$ form at this position, while $90 \%$ contained the standard Y form of the base (Table S18). However, no Y form was observed in any ladder fragments containing this position after acid degradation, and all of the Y bases were converted to $\mathrm{Y}^{\prime}$ due to depurination with the acidic conditions (Fig. 2A). As another piece of evidence of the depurination, a mass of $376.1178 \mathrm{Da}$, corresponding to a cleaved $\mathrm{Y}$ nucleobase, was found in the crude products after acid degradation and subsequent MS analysis (Fig. 2B), suggesting that Y was originally carried by the tRNA. The fact that our method can identify the dynamic change of $\mathrm{Y}$ to $\mathrm{Y}^{\prime}$ and quantify the relative $\mathrm{Y} / \mathrm{Y}^{\prime}$ ratio could be useful for clinical assays. Certain cancer cells have an acidic $\mathrm{pH}(20)$, and acid-mediated conversion of $Y$ to $\mathrm{Y}^{\prime}$ could potentially occur in this $\mathrm{pH}$ regime within these cells (21). Such changes in the $\mathrm{Y}^{\prime} / \mathrm{Y}$ ratio could be used as a potential biomarker for cancer diagnoses. Based on the same principle, our method could potentially probe dynamic changes of other base modifications, and quantify variations in their ratios in particular cells or tissues subjected to different biological processes.

Unlike its nominal identity according to the supplier, upon sequencing, the commercially-prepared tRNA $^{\text {Phe }}$ sample was revealed to be heterogeneous. Besides the 76 nt tRNA with a posttranscriptionally modified CCA tail, two other isoforms of the tRNA that are missing an A and a CA at the 3'-CCA tail, respectively, were identified in a $3^{\prime}$ segment of the tRNA (58m $\left.{ }^{1} \mathrm{~A}-76 \mathrm{~A}\right)$ (Fig.1B and Fig. S6) using the anchor algorithms and a revised Smith-Waterman alignment similarity algorithm (details in Supplementary Materials). Surprisingly, the most abundant component was not the nominal 76 nt tRNA ${ }^{\text {Phe }}$, which comprised only $17 \%$ of the sample as measured by integration of the corresponding extracted-ion currents (EIC) (Fig. S6C) (11). Rather the $75 \mathrm{nt}^{\mathrm{tRNA}}{ }^{\text {Phe }}$ with a missing A at the $3^{\prime}$ end was the major component of the sample, at $80 \%$, while the $74 \mathrm{nt}$ tRNA ${ }^{\text {Phe }}$ with a missing $\mathrm{CA}$ at the $3^{\prime}$ end was a minor component at $3 \%$. The two tail-truncation isoforms cannot be degraded products of longer tRNAs like the $76 \mathrm{nt}^{\mathrm{tRNA}}{ }^{\text {Phe }}$, otherwise, they would not have the free 3 ' $-\mathrm{OH}$ required for the 2D HELS chemistry (15). The data suggest that 2-D HELS MS Seq is not only is able to sequence modified RNA, it can also identify tail-truncation isoforms that were primarily only studied by polyacrylamide gel electrophoresis methods previously (22). As stress-induced tRNA truncation has been implicated in cancers and other diseases (23), further studies into the relationship between the relative abundances of tRNA $^{\text {Phe }}$ tail-truncation isoforms and various diseases will assist in understanding the role of such isoforms in disease-related biological processes and subsequent treatments (24).

Finally, we also observed two isoforms with an A to $\mathrm{G}$ transition at position 44 and a $\mathrm{G}$ to $\mathrm{A}$ transition at position 45, i.e., 44A45G (wild type, reported previously) (25) to 44g45a transition 
(Lower case $\mathrm{g}$ and a are used to differentiate them from the canonical $\mathrm{G}$ and $\mathrm{A}$ in positions 44 and 45 , respectively). These two reads were revealed first by our algorithm, and verified manually in the original MFE files (Fig. 3, Tables S4-5, S8-9, and S19-22). We identified two distinct mass ladder fragments at position 44 when reading from the 5 ' direction, apparently corresponding to sequences containing both $44 \mathrm{~A}$ and $44 \mathrm{~g}$ being simultaneously present. However, these two mass ladder fragments merged into one mass ladder fragment at position 45. Such an effect could only occur if two co-existing sequences contained a $45 \mathrm{G}$ or a $45 \mathrm{a}$, respectively, thus confirming the coexistence of two co-existing isoforms (Fig. 3A). This is consistent with the sequencing results when reading from the opposite direction when we performed bi-directional sequencing (14) (Fig. 3C). We observed these two isoforms in all reads which covered positions 44 and 45, and their relative percentages were consistent ( $\sim 50 \%$ for wild-type, quantified by EIC) (Table S23). To further verify the co-existence of the two mass fragments, we employed full-spectral analysis provided by commercial MassWorks software (Cerno Bioscience, USA) to examine the corresponding ions of these two fragments simultaneously in one spectrum. When reading from the $5^{\prime}$ direction, two ions (m/z 778.1051 and 779.7068, both with 10 charge states) were found, corresponding to $44 \mathrm{~A}$ and $44 \mathrm{~g}$. Full-spectral analysis also confirmed that $45 \mathrm{G}$ and $45 \mathrm{a}$ co-exist when reading from the $3^{\prime}$ direction (Fig. 3D). Furthermore, the ratios of $44 \mathrm{~A} / 44 \mathrm{~g}$ as compared to $45 \mathrm{G} / 45 \mathrm{a}$ quantified by the full-spectral analysis (26) are consistent (Fig. 3), indicating that the sequenced $44 \mathrm{~g}$ and the $45 \mathrm{a}$ are indeed from the same RNA strand, while the $44 \mathrm{~A}$ and $45 \mathrm{G}$ are also both from the same RNA strand. All these MS results support the existence of an isoform, with the sequence $44 \mathrm{~g} 45 \mathrm{a}$, co-existing with the wild-type RNA that contains the 44A45G sequence, and that these two isoforms occur at similar levels.

To further confirm the co-existence of these isoforms, we performed a reverse transcription single base extension (rtSBE) on the tRNA ${ }^{\text {Phe }}$ sample. (The rtSBE method was also used in this study to previously differentiate between $\mathrm{m}^{1} \mathrm{~A}$ and $\mathrm{m}^{6} \mathrm{~A}$ (Fig. S3)). For example, if tRNA ${ }^{\text {Phe }}$ has an A/g SNP at position 44, then the rtSBE assay would be able to incorporate both ddT and $\mathrm{ddC}$, since the two isoforms exist at similar levels. However, the results showed that only ddT could be incorporated at position 44 (Fig. S7) and only ddC could be incorporated at position 45 (Fig. S7), indicating that the wildtype 44A45G was the only isoform present. The rtSBE results suggested that RNA reverse transcriptase cannot well recognize these edited bases. It is also possible that the mass differences observed in the above A-G transition at position 44 may be caused by oxidation and reduction, e.g., oxidation of A to isoG and 8-oxoA (Fig. S8A), which both have a mass identical to G. Complete acid digestion of the tRNA into single nucleotides followed by LC-MS analysis support this possibility, as we found two different $t_{R S}$ in the EIC profile of the G monophosphate (Fig. S8B), suggesting a co-existing nucleotide of the same mass as $\mathrm{G}$, but a different structure. A similar mechanism could explain the putative $G$ to A transition at position 45. Regardless, the results of the 2D-HELS-AA MS Seq revealed new isoforms, RNA base modifications and editing as well as their relative abundance in the tRNA that can't be determined by cDNA-based methods (Fig. 4), opening new opportunities in the field of epitranscriptomics.

Acknowledgments: The authors acknowledge the R21 grant from NIH (R21HG009576) to S. Z. and W. L. and New York Institute of Technology (NYIT) Institutional Support for Research and Creativity grants to S. Z., which supported this work. The authors would also like to thank $\mathrm{PhD}$ student Xuanting Wang (Columbia University) for assisting in figure-making, and Prof. Michael 
Hadjiargyrou (NYIT), Prof. Jingyue Ju (Columbia University), Drs. Shiv Kumar, Xiaoxu Li, Steffen Jockusch and other members of the Ju lab (Columbia University), Dr. Yongdong Wang (Cerno Bioscience), and Meina Aziz (NYIT) for helpful discussions and suggestions for our manuscript.

Funding: National Institutes of Health R21 Grant [R21HG009576 to S. Z., W. L.]; New York Institute of Technology Institutional Support for Research and Creativity grants (to S. Z.); World Premier International Research Center Initiative grant from the Japanese Ministry of Education, Culture, Sports, Science and Technology (to T.Z.J. via the Earth-Life Science Institute at Tokyo Institute of Technology).

Author contributions: N.Z., S.S., X.W. W.N., T.Z.J., J.J.R., and A.Z. performed experiments and data analysis. B.Y. performed mass spectrometry data acquisition. X.Y., D.J., and W.L. developed anchor-based sequence algorithms, while N.Z., S.S., and S.Z. wrote the manuscript. The original idea for this manuscript was conceived by S.Z.

Competing interests: The authors have filed a provisional patent related to the technology discussed in this manuscript.

Data and materials availability: All data is available in the main text or the supplementary materials. Anchor-based algorithms are available upon request. 


\section{Figures and Figure Legends}

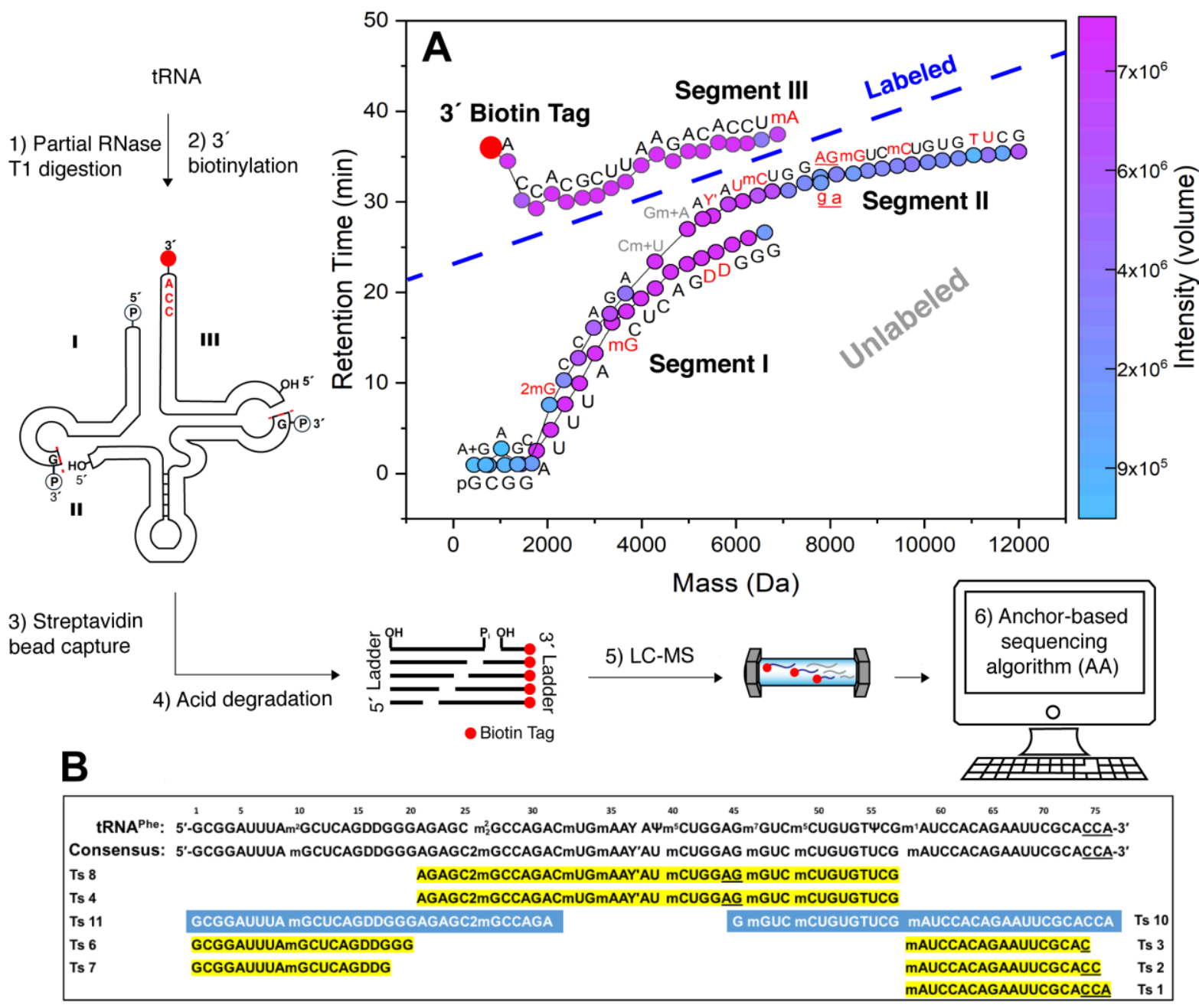

Fig.1. 2D-HELS-AA MS Seq of Yeast tRNA ${ }^{\text {Phe }}$. 1-6: Sequencing workflow. More detailed information can be found in Materials and Methods (supplementary materials). A. The 2D plot of the entire tRNA sequenced from a single LCMS run, showing the identity and location of all the modifications. B. Assembly of the full-length tRNA ${ }^{\text {Phe }}$ sequence based on overlapping sequence reads from different LC-MS runs, showing 100\% coverage and accuracy as compared to the reported tRNA ${ }^{\text {Phe }}$ reference sequence. All output sequence reads are converted to FASTA format in the $5^{\prime}$ to $3^{\prime}$ order (44 and 45 AG conversion output reads not included). *Ts: the Supplementary Table $\mathrm{S}$ where the sequencing data of that particular strand can be found. 
A

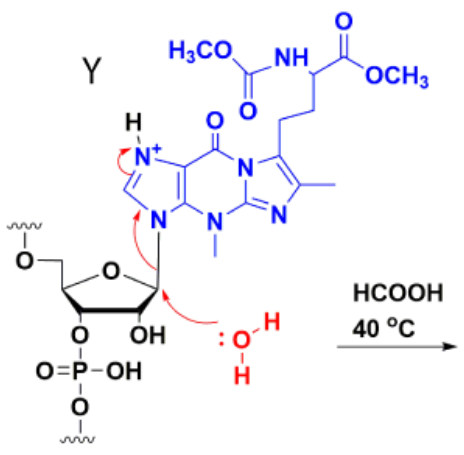

B

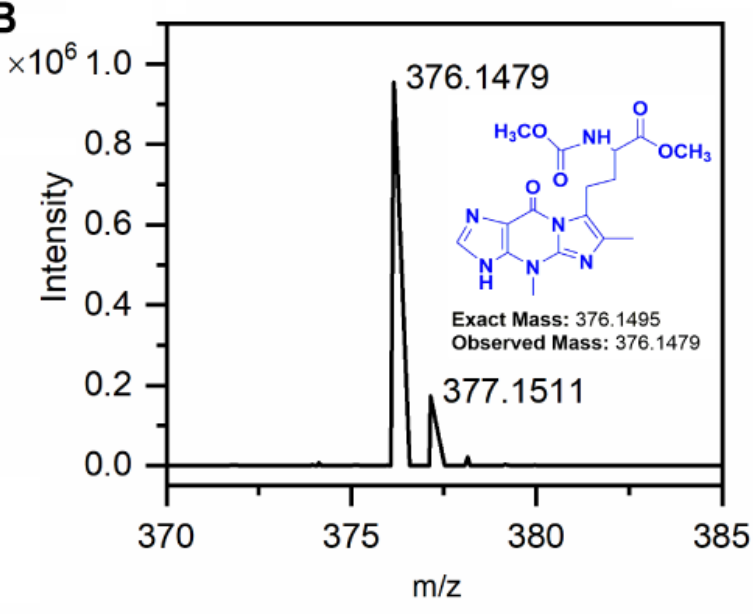

85

C

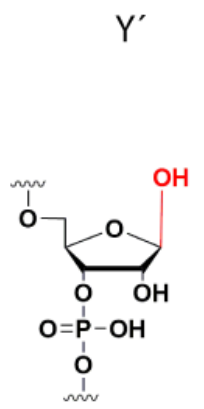

\begin{tabular}{|c|c|c|c|c|c|c|}
\hline$\#$ & Common Name & Algorithm Symbol & Position & Detection Method & $\mathrm{SI}$ & $\%$ \\
\hline 1 & dihydrouridine (D) & $\mathrm{D}$ & 16,17 & By unique mass & - & 100 \\
\hline 2 & $\mathrm{~N}^{2}, \mathrm{~N}^{2}$ dimethylguanosine $\quad\left(\mathrm{m}_{2}^{2} \mathrm{G}\right)$ & $2 m G$ & 26 & By unique mass & - & $\mathrm{m}_{2}^{2} \mathrm{G}: \mathrm{mG}: \mathrm{G}=58: 2.6: 39.4$ \\
\hline 3 & 5-methylcytidine $\left(\mathrm{m}^{5} \mathrm{C}\right)$ & $\mathrm{mC}$ & 40,49 & By unique mass & - & 100 \\
\hline 4 & 5-methyluridine $(\mathrm{T})$ & $\mathrm{T}$ & 54 & By unique mass & - & 100 \\
\hline 5 & 2'-O-methylcytidine $\left(C_{m}\right)$ & $C_{m}$ & 32 & MS/MS & Fig. 52 & 100 \\
\hline 6 & 2'-O-methylguanosine $\left(G_{m}\right)$ & $\mathrm{G}_{\mathrm{m}}$ & 34 & MS/MS & Fig. 52 & $\mathrm{Gm:} \mathrm{G}=66.9: 33.1$ \\
\hline 7 & 1-methyladenosine $\left(m^{1} A\right)$ & $\mathrm{mA}$ & 58 & rtSBE ( -) & Fig. 53 & $\mathrm{~m}^{\prime} \mathrm{A}: \mathrm{A}=96.1: 3.9$ \\
\hline 8 & pseudouridine $(\boldsymbol{\Psi})$ & $u$ & 39,55 & CMC conversion (+) & Fig. 54 & 100 \\
\hline 9 & $\mathrm{~N}^{2}$-methylguanosine $\left(\mathrm{m}^{2} \mathrm{G}\right)$ & $\mathrm{mG}$ & 10 & Aniline cleavage ( -) & Fig. 55 & 100 \\
\hline 10 & 7-methylguanosine $\left(\mathrm{m}^{7} \mathrm{G}\right)$ & $\mathrm{mG}$ & 46 & Aniline cleavage (+) & Fig. 55 & $\mathrm{~m}^{7} \mathrm{G}: \mathrm{G}=56.2: 47.8$ \\
\hline \multirow{2}{*}{11} & wybutosine $(\mathrm{Y})$ & $Y$ & \multirow{2}{*}{37} & \multirow{2}{*}{$\begin{array}{c}\text { Conversion to } \mathrm{Y} \text { ' under } \\
\text { acid condition }\end{array}$} & \multirow{2}{*}{ Fig. $2 \mathrm{~A}$} & 90 \\
\hline & ribose $\left(Y^{\prime}\right)$ & $Y^{\prime}$ & & & & 10 \\
\hline
\end{tabular}

Fig. 2. Identification and the subsequent quantification of a dynamic change from wybutosine (Y) to its depurinated $\mathbf{Y}^{\prime}$ form in tRNA ${ }^{\text {Phe }}$. A. A proposed mechanism for the conversion of the $Y$-base to $\mathrm{Y}^{\prime}$ in acidic conditions. B. The mass of nucleobase $\mathrm{Y}$ was found in the crude products after acid degradation. The relative percentages of $\mathrm{Y}$ and $\mathrm{Y}^{\prime}$ were quantified and can be found in Table S18. C. Summary of all 11 RNA modifications sequenced by 2D-HELS-AA MS Seq. The relative percentages of modifications at each position were quantified by EIC of their corresponding ladder fragments. 
A
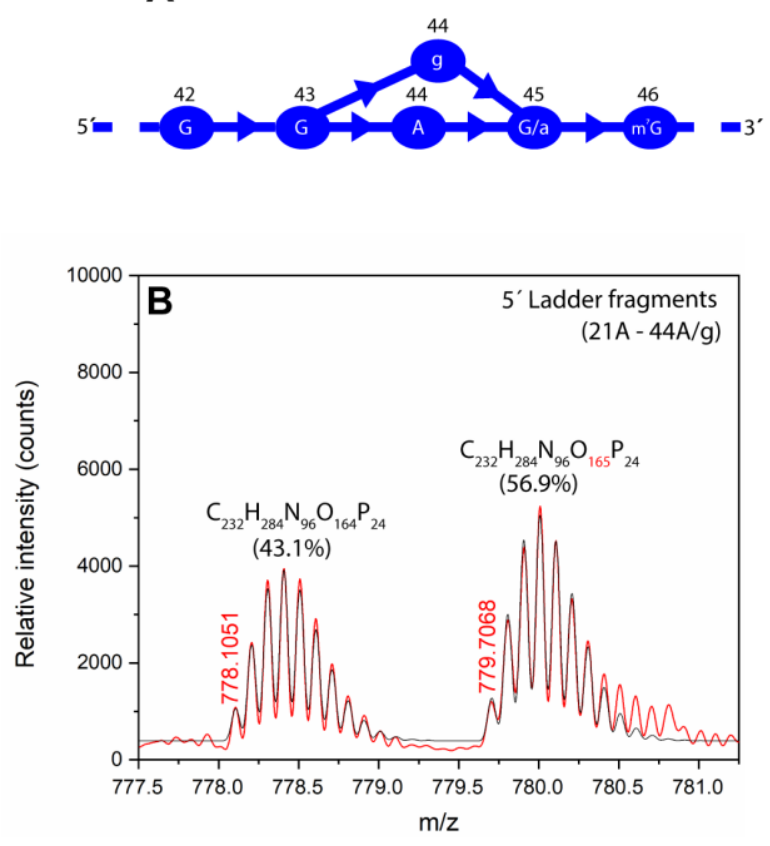

C
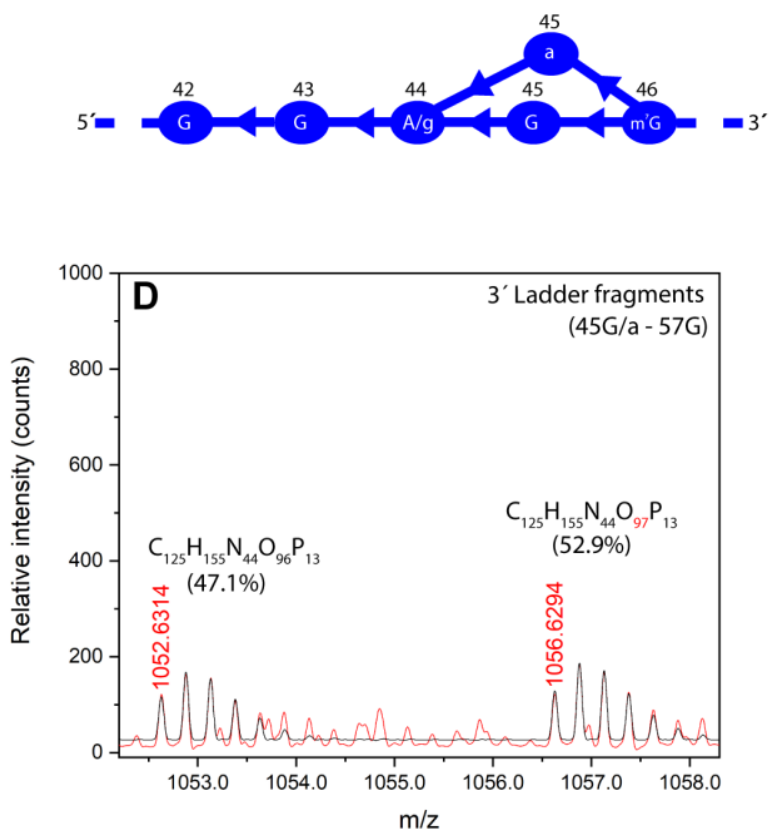

Fig. 3. LC-MS results of $44 \mathrm{~A} 45 \mathrm{G}$ to $44 \mathrm{~g} 45 \mathrm{a}$ transitions at variable region of tRNA. A. A schematic picture of sequence ladder fragments shows a transition $\mathrm{g}$ (sharing an identical mass as $\mathrm{G}$ ) co-exists with A at position 44 when reading from the 5' direction (Tables S4-5 and S8-9). B. Least squares fitted mass spectrum to the calibrated mass spectrum $\left(t_{R}=31.9-32.9 \mathrm{~min}\right)$ when reading from the $5^{\prime}$ direction. The full-spectral analysis confirms that the ions of the $44 \mathrm{~g}$ and $44 \mathrm{~A}$ fragments (with 10 charges) co-exist and that their relative abundances are $57 \%$ and $43 \%$, respectively. The theoretical trace (black) of the two combined ion profiles fits well with the calibrated mass spectrum as observed (red), resulting in a good spectral accuracy of $87 \%$. C. A single transition a (one oxygen less than G) coexists with $\mathrm{G}$ at position 45 when reading from the 3' direction (Tables S19-22). D. Similarly to B, full-spectral analysis confirms that the ions of the 45a and 45G fragments (both with 4 charges; spectral accuracy: 71\%) also coexist and their relative abundances are $47 \%$ and $53 \%$, respectively, when reading from the $3^{\prime}$ direction $\left(t_{R}=16.5-18.6\right.$ $\min )$. 


\begin{tabular}{ccc}
\hline Symbol & Position & $\%$ \\
\hline $\mathrm{m}^{2} \mathrm{G}$ & 10 & 100 \\
$\mathrm{D}$ & 16,17 & 100 \\
$\mathrm{~m}^{2} \mathrm{G}$ & 26 & 58.2 \\
$\mathrm{C}_{\mathrm{m}}$ & 32 & 100 \\
$\mathrm{G}_{\mathrm{m}}$ & 34 & 66.9 \\
$\mathrm{Y}$ & 37 & 90 \\
$\boldsymbol{\Psi}$ & 39,55 & 100 \\
$\mathrm{~m}^{5} \mathrm{C}$ & 40,49 & 100 \\
$\mathrm{~m}^{7} \mathrm{G}$ & 46 & 56.2 \\
$\mathrm{~T}$ & 54 & 100 \\
$\mathrm{~m}^{1} \mathrm{~A}$ & 58 & 96.1 \\
\hline
\end{tabular}

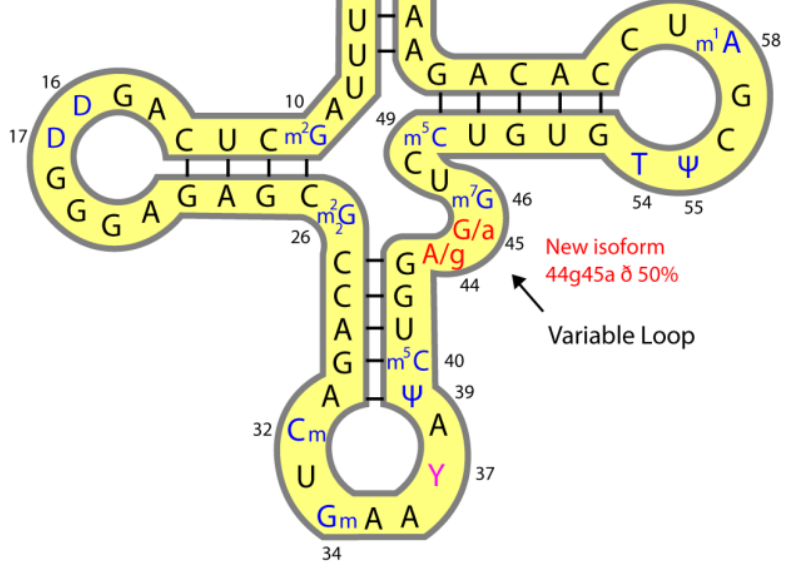

Fig. 4. Summary of different isoforms, RNA base modifications and editing as well as their relative abundance in the tRNA ${ }^{\text {phe }}$. 


\section{References:}

1. C. Lorenz, C. E. Lunse, M. Morl, tRNA Modifications: Impact on Structure and Thermal Adaptation. Biomolecules 7, (2017).

2. C. Bou-Nader et al., Unveiling structural and functional divergences of bacterial tRNA dihydrouridine synthases: perspectives on the evolution scenario. Nucleic Acids Res 46, 1386-1394 (2018).

3. R. W. Holley et al., Structure of a Ribonucleic Acid. Science 147, 1462-1465 (1965).

4. G. Zheng et al., Efficient and quantitative high-throughput tRNA sequencing. Nat Methods 12, 835-837 (2015).

5. U. L. RajBhandary, C. Kohrer, Early days of tRNA research: discovery, function, purification and sequence analysis. J Biosci 31, 439-451 (2006).

6. T. Carell et al., Structure and function of noncanonical nucleobases. Angew Chem Int Ed Engl 51, 71107131 (2012).

7. S. Kellner, J. Burhenne, M. Helm, Detection of RNA modifications. RNA Biol 7, 237-247 (2010).

8. $\quad$ F. Ozsolak et al., Direct RNA sequencing. Nature 461, 814-818 (2009).

9. D. R. Garalde et al., Highly parallel direct RNA sequencing on an array of nanopores. Nat Methods 15, 201-206 (2018).

10. M. Taucher, K. Breuker, Characterization of modified RNA by top-down mass spectrometry. Angew Chem Int Ed Engl 51, 11289-11292 (2012).

11. T. Y. Huang, J. Liu, S. A. McLuckey, Top-down tandem mass spectrometry of tRNA via ion trap collisioninduced dissociation. J Am Soc Mass Spectrom 21, 890-898 (2010).

12. T. Suzuki, T. Suzuki, A complete landscape of post-transcriptional modifications in mammalian mitochondrial tRNAs. Nucleic Acids Res 42, 7346-7357 (2014).

13. B. Thomas, A. V. Akoulitchev, Mass spectrometry of RNA. Trends Biochem Sci 31, 173-181 (2006).

14. A. Bjorkbom et al., Bidirectional direct sequencing of noncanonical RNA by two-dimensional analysis of mass chromatograms. J Am Chem Soc 137, 14430-14438 (2015).

15. N. Zhang et al., A general LC-MS-based RNA sequencing method for direct analysis of multiple-base modifications in RNA mixtures. Nucleic Acids Research gkz731, https://doi.org/10.1093/nar/gkz731 (2019).

16. Z. Chen et al., Transfer RNA demethylase ALKBH3 promotes cancer progression via induction of tRNAderived small RNAs. Nucleic Acids Res 47, 2533-2545 (2019).

17. V. Marchand et al., AlkAniline-Seq: Profiling of m(7) G and m(3) C RNA Modifications at Single Nucleotide Resolution. Angew Chem Int Ed Engl 57, 16785-16790 (2018).

18. U. L. RajBhandary, R. D. Faulkner, A. Stuart, Studies on polynucleotides. LXXIX. Yeast phenylalanine transfer ribonucleic acid: products obtained by degradation with pancreatic ribonuclease. J Biol Chem $\mathbf{2 4 3}$, 575-583 (1968).

19. J. E. Ladner, M. P. Schweizer, Effects of dilute $\mathrm{HCl}$ on yeast tRNAPhe and E. coli tRNA1fMet. Nucleic Acids Res 1, 183-192 (1974).

20. R. A. Gatenby, E. T. Gawlinski, A. F. Gmitro, B. Kaylor, R. J. Gillies, Acid-mediated tumor invasion: a multidisciplinary study. Cancer Res 66, 5216-5223 (2006).

21. R. Thiebe, H. G. Zachau, A specific modification next to the anticodon of phenylalanine transfer ribonucleic acid. Eur J Biochem 5, 546-555 (1968).

22. C. Merryman, E. Weinstein, S. F. Wnuk, D. P. Bartel, A bifunctional tRNA for in vitro selection. Chem Biol 9, 741-746 (2002).

23. D. M. Thompson, R. Parker, Stressing Out over tRNA Cleavage. Cell 138, 215-219 (2009).

24. Y. M. Hou, CCA addition to tRNA: implications for tRNA quality control. IUBMB Life 62, 251-260 (2010).

25. B. Alzner-DeWeerd, L. I. Hecker, W. E. Barnett, U. L. RajBhandary, The nucleotide sequence of phenylalanine tRNA from the cytoplasm of Neurospora crassa. Nucleic Acids Res 8, 1023-1032 (1980).

26. Y. Wang, M. Gu, The concept of spectral accuracy for MS. Anal Chem 82, 7055-7062 (2010). 